\title{
Über den Formylglutaconsäureester und seine Isomerieverhältnisse;
}

\section{von Wilhelm Wislicenus und Margarita von Wrangell.}

(Mitteilung aus dem chemischen Laboratorium der Universität Tübingen.)

(Eingelaufen am 21. April 1911.)

Der Formyl- oder Oxymethylenglutaconsäureester ${ }^{1}$ )<smiles>CCOC(=O)C(C=CCO)C=CCCCC(=O)OC</smiles>

entsteht aus dem Formylessigester, indem sich 2 Mol. des letzteren unter Wasseranstritt kondensieren. Aus Äther oder Benzol krystallisiert er in farblosen Blättchen, die bei $66-67^{\circ}$ schmelzen und in Alkohol eine intensive blauviolette Eisenchloridreaktion geben. Beim Aufbewahren an feuchter Luft und beim Schmelzen geht er langsam in eine zähölige Form über, deren Eisenchloridreaktion rot ist, und aus der beim Erhitzen Trimesinsäureester entsteht. $\left.{ }^{2}\right)$

Es hat sich nun gezeigt, daß diese ölige Form die gleiche Zusammensetzung, aber das doppelte Molekulargewicht wie die krystallisierte Form besitzt und mithin nach der Formel $\mathrm{C}_{20} \mathrm{H}_{28} \mathrm{O}_{10}$ zusammengesetzt ist.

Abgesehen von den bereits aufgezählten Gelegenheiten zur Polymerisation wandelt sich der Formylglutaconsäureester auch in Lösung in das bimolekulare Öl um. Die Geschwindigkeit dieses Vorgangs ist aber ungemein verschieden je nach der Natur des Lösungs-

1) Diese Annalen 316, 20 (1901); 356, 32 (1907). Wir wählen im folgenden für den Ester die Benennung „Formylglutaconsäureester", nicht nur wegen der Kürze, sondern auch, weil er dadurch als Kondensationsprodukt des Ameisensäureesters gekennzeichnet ist. Die allein isolierbare Form besitzt aber jedenfalls die Enolstruktor und enthält somit die "Oxymethylen"-Gruppe.

2) Diese Annalen 316, 30 (1901). 
mittels. Es ist schon früher angegeben worden, daß Benzol geradezu ein Konservierungsmittel für die monomolekulare krystallisierte (Enol-)Form ist. ${ }^{1}$ ) Eine solche Lösung gibt selbst nach langem Stehen noch die charakteristische violette Eisenchloridreaktion und beim $\mathrm{Ab}$ dunsten die Krystallblättchen wie in frischem Zustande. Zufällig war aber diese Lösung noch weitere Jahre stehen geblieben und es zeigte sich, dab endlich doch auch hier die Polymerisation eingetreten war: Der Verdunstungsrückstand war ölig und gab die rote Eisenchloridreaktion. Es ist denkbar, daß allmählich zudringende Feuchtigkeit diesen Übergang veranlaßt hat. Jedenfalls tritt in Benzol die Polymerisation nur äußerst langsam, vielleicht an sich überhaupt nicht ein.

In absolutem Methylalkohol bleibt die violette Eisenchloridreaktion längere Zeit bestehen, wird aber schwächer. Diese Abnahme der Intensität, die durch das Colorimeter bestimmt war, ist in $1 / 10$ molekularer Lösung sehr beträchtlich und läßt auf ein Gleichgewicht zwischen der eisenbläuenden Enolform und einer gegen Eisenchlorid reaktionslosen Aldoform schließen:

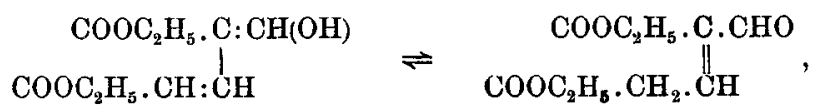

welch letztere in der alkoholischen Lösung gebildet wird.

Dunstet man eine solche Lösung bei gewöhnlicher Temperatur ein, so erhält man manchmal die Krystalle der Enolform zurück, meist aber ist der Rückstand in die ölige bimere Form mit der roten Eisenchloridreaktion übergegangen. Die Bedingungen, unter denen man das eine oder das andere Resultat erhält, sind noch nicht ermittelt.

Rasch und vollständig erfolgt die Polymerisation in einer Lösung von feuchtem Äther. Bei gewöhnlicher Temperatur ist die Reaktion in ein bis zwei Tagen vollzogen. Eine Probe, mit Alkohol und Eisenchlorid versetzt,

1) Diese Annalen 316, 30 (1901). 
zeigt dann nicht mehr die violette, sondern die rote Färbung und hinterläßt beim Abdunsten die ölige Form. Setzt man dagegen dem feuchten Äther einen Tropfen verdünnte Schwefelsäure oder Salzsäure zu, so tritt diese Veränderung auch in viel längerer Zeit nicht ein. Säure verhindert also die Polymerisation.

Wie beim Formylglutaconsäureester ist auch beim Formylfluoren ${ }^{1}$ ) die Umwandlung in ein Isomeres mit doppeltem Molekulargewicht beobachtet worden. Diesen Formylverbindungen reiht sich der von Claisen ${ }^{2}$ ) entdeckte Mesityloxydoxalester an. Bei einer Untersuchung über die Veränderungen dieser Substanz in Lösungen, welche Federlin ${ }^{3}$ ) auf die Veranlassung des einen von uns ausgeführt hat, wurde ebenfalls die Entstehung einer in diesem Falle gut krystallisierenden dimeren Form nachgewiesen.

Die Reaktionsfähigkeit des bimolekularen Formylglutaconsäureesters steht weit hinter der des einfachen Esters zurück. Es gelang z. B. nicht, ein Brom-, ein Benzoyl- oder ein Phenylhydrazinderivat darzustellen, Substanzen, die aus dem gewöhnlichen Formylglutaconsäureester leicht entstehen. Dagegen scheint ein Phenylisocyanatadditionsprodukt $\mathrm{C}_{20} \mathrm{H}_{28} \mathrm{O}_{10} \cdot \mathrm{C}_{7} \mathrm{H}_{5} \mathrm{ON}$ zu existieren. Diese Substanz ist bisher nur als ein undestillierbares öl erhalten worden. Wenn man sie indessen aus der Ätherlösung durch Petroläther, in welchem sie unlöslich ist, ausfällt, so ist sie genügend rein, um die auf die angegebene Formel stimmenden Analysenwerte zu liefern.

Daraus und aus der roten Eisenchloridreaktion ist zu schließen, daß bei der Aneinanderlagerung zweier Moleküle des einfachen Formylglutaconsäureesters die eine Formyl- bzw. Oxymethylengruppe erhalten geblieben ist, während die andere sich verändert hat.

1) Ber. d. d. chem. Ges. 43, 2719 (1910).

2) Diese Annalen 291, 111 (1896).

3) Diese Annalen 356, 251 (1907). 
Einen weiteren Anhaltspunkt über den Verlauf des Polymerisationsvorganges kann man gewinnen, wenn man das Verhalten in Lösung berücksichtigt. Benzol begünstigt die Enolform der Formylgruppe und verzögert oder verhindert die Polymerisation; in Methylalkohol findet eine teilweise Umlagerung in die Aldoform statt und beim Verdunsten entsteht meist das bimolekulare Öl; feuchter Äther bewirkt durch seinen Wassergehalt eine rasche Polymerisierung. Dieser Einfluß des Lösungsmittels auf die Verdoppelung des Moleküls ist danach offenbar ein indirekter, zunächst bewirken die stark dissoziierenden Medien eine desmotrope Umlagerung in die Aldoform, und erst diese ist dann imstande, ein zweites Molekül anzulagern. Gestützt wird diese Auffassung dadurch, daß etwas ganz Analoges beim Mesityloxydoxalester bereits bewiesen ist. ${ }^{1}$ ) Dort polymerisiert sich direkt nur die Ketoform, die Enolform erst, nachdem sie sich in alkoholischen Lösungen in die erstere umgelagert hat. In Benzol oder Chloroform, worin die Enolform beständig ist, tritt die Polymerisation entweder gar nicht oder äußerst langsam ein.

Es scheint also, als ob nur eine echte Aldehydgruppe befähigt sei, ein zweites Molekül des Esters anzulagern. Ist es gestattet auf solche Überlegungen eine Formel zu gründen, so könnte man sich folgendes Bild machen:

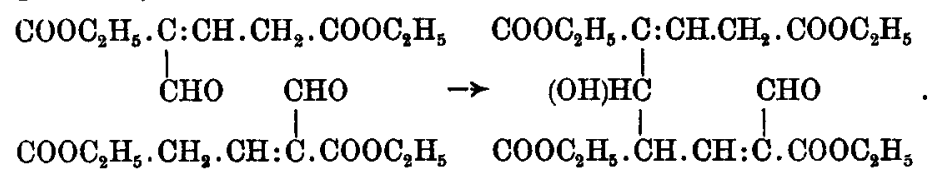
polymerer Formylglutaconester ${ }^{2}$ )

Wir würden uns mit der Aufstellung dieser Formel nicht so weit von dem Beweisbaren entfernt haben, wenn es nicht wiederum eine Reaktion gäbe, die im besten Einklang damit stände. Das ist der bei $120^{\circ}$ erfolgende

1) Diese Annalen 356, $266 \mathrm{f}$. (1907).

2) Oder eine tautomere Formel mit der sauren Oxymethylengruppe. 
Formylglutaconsäureester und seine Isomerieverhältnisse. 371

fast quantitativ verlaufende Zerfall des dimeren Formylglutaconsäureesters in Trimesinsäureester, Ameisensäure und Essigester, den man in folgender Weise wiedergeben kann:

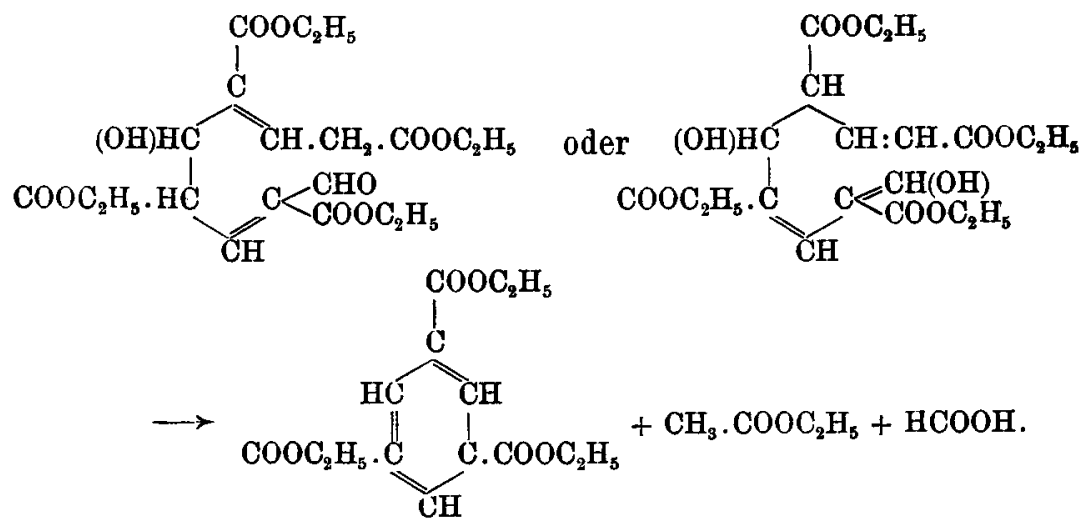

Dem Trimesinsäureester begegnet man beim Arbeiten mit Formylessigester und Formylglutaconsäureester immer wieder. Es soll aber nicht behauptet werden, dab er immer auf diesem Umweg über den dimeren Ester entsteht. Es erscheint vielmehr als wahrscheinlich, daß sich der Frormylessigester $\mathrm{CH}(\mathrm{OH}): \mathrm{CH} . \mathrm{COOC}_{2} \mathrm{H}_{5}$, sowie er durch Ansäuern aus seiner Natriumverbindung in Freiheit gesetzt ist, in zweierlei Weise kondensieren kann: Einmal entsteht aus $2 \mathrm{Mol}$. unter Wasseraustritt der Formylglutaconsänreester, das andere Mal aus 3 Mol. direkt der Trimesinsäureester, wie es schon früher von Piutti ${ }^{1}$ ) angenommen worden ist.

Aus dem zuerst gebildeten Formylglutaconsäureester kann auf dem geschilderten Wege abermals Trimesinsäureester entstehen und es scheint hierbei die Gegenwart von Alkali begünstigend zu wirken. V.Pechmann ${ }^{2}$ ) hat die Beobachtung gemacht, daß sich aus wäßrigalkalischen Lösungen des Formylglutaconsäure-methylesters beim Stehen allmählich Trimesinsäuremethylester

1) Ber. d. d. chem. Ges. 20, 538 (1887).

2) Diese Annalen 273, 175 (1893). 
abscheidet. Beim Äthylester konnte die gleiche Reaktion bemerkt werden, als Kohlendioxyd in die alkalische Lösung eingeleitet wurde.

Auffallend ist auch folgende Beobachtung: Wenn eine ätherische Lösung von Formylglutaconsäureester mit einer alkoholisch-ätherischen Lösung von Kaliumäthylat in einem solchen Mengenverhältnis vermischt wird, dab nur ein Teil des Esters in die farblose Kaliumverbindung übergehen kann, so verwandelt sich der Rest bei längerem Stehen in Trimesinsäureester, der sich zwischen dem mikrokrystallinischen Niederschlag der Kaliumverbindung in Form derber zweispitziger skalenoederähnlicher Krystalle ausscheidet.

Es gibt also beim Arbeiten mit Formylessig- und Formylglutaconsäureester sehr mannigfaltige Gelegenheit zur Bildung des Trimesinsäureesters und es ist deshalb leicht erklärlich, daß man ihn in allen Mutterlaugen nach einiger Zeit vorfindet. Er erscheint für gewöhnlich in Form schöner farbloser Nädelchen vom Schmelzp. 133 bis $134^{\circ}$.

Der krystallisierte monomolekulare Formylglutaconsäureester addiert Chlor und Brom in molekularem Verhältnis. Die Additionsprodukte verhalten sich aber sehr verschieden. Die Chlorverbindung ist ein Öl, welches keine Eisenchloridreaktion mehr zeigt. Beim Erhitzen spaltet sie Alkohol ab und es ist wahrscheinlich, dab hierbei Dichlordihydrocumalinsäureester entsteht:

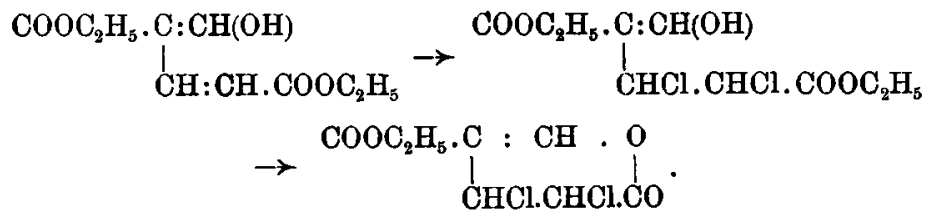

Etwas anders ist das Verhalten der gleichfalls öligen Bromverbindung, welche zuerst Bromwasserstoff und dann erst beim Erhitzen Alkohol abspaltet. Ein Verlust an Bromwasserstoff tritt langsam bereits ein, wenn 
man das ÖI in einem Exsiccator neben Kali stehen läßt. Hierbei ist es einigemal gelungen, ein öliges Derivat von der Zusammensetzung $\mathrm{C}_{10} \mathrm{H}_{13} \mathrm{O}_{5} \mathrm{Br}$ und roter Eisenchloridreaktion zu erhalten. Unter Umständen, die wir nicht sicher in der Hand hatten, ließen sich kleine Mengen sogar im Vakuum destillieren. Leicht und sicher läßt sich dagegen ein krystallisierendes Isomeres mit violetter Eisenchloridreaktion darstellen, wenn man das ölige Additionsprodukt mit einer alkoholisch-ätherischen Lösung von Kaliumäthylat in molekularer Menge behandelt. Es scheidet sich Kaliumbromid ab und beim Findunsten der Mutterlauge hinterbleiben Krystalle vom Schmelzp. $56^{\circ}$, die sich als Bromformylglntaconsäureester erwiesen haben. Die geometrische Konfiguration dieses Esters ist dadurch festgestellt, daß er über $100^{\circ}$, am schnellsten bei der Vakuumdestillation Alkohol verliert und in Bromcumalinsäureester (Schmelzp.94-950) übergeht ${ }^{1}$ ):

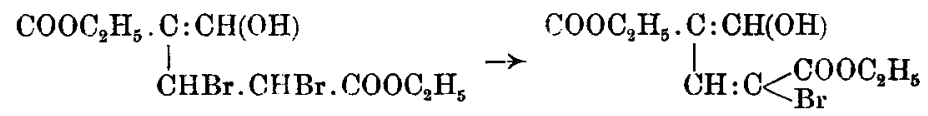

Bromformylglutaconsäure-

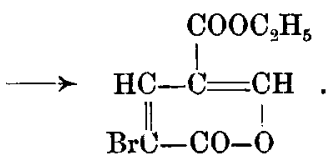
ester

Bromcumalinsäureester

Der krystallisierte Bromformylglutaconsäureester bildet sich auch aus dem Additionsprodukt bei längerem Stehen neben Kali, oder - in Form seiner Kupfer-

1) Die oben beschriebene ölige Substanz mit der roten Eisenchloridreaktion ist möglicherweise ein geometrisch isomerer Bromformylglutaconsäureester:<smiles>CCOC(=O)C(Br)C(O)C(CC)C(=O)OCC</smiles>

Da die Bedingungen ihrer Entstehung bisher nicht mit Sicherheit gefunden worden sind, muß die Entscheidung über die Richtigkeit dieser Aunahme einstweilen ausgesetzt werden. 
verbindung -, wenn man das Bromadditionsprodukt in Chloroformlösung anhaltend und wiederholt mit Kupferacetatlösung schüttelt.

Der Bromcumalinsäureäthylester ist bisher noch unbekannt gewesen. Dagegen hat v. Pechmann ${ }^{1}$ ) den analogen Methylester - von der Cumalinsäure ausgehend - auf zwei Wegen erhalten. Die $\alpha$-Stellung des Broms und damit die Konstitution des Pechmannschen Esters hat Feist ${ }^{2}$ ) festgestellt, indem er zeigte, daß durch kochende Kalilauge Furfuran-2,4-dicarbonsäure gebildet wird. Da es gelang, durch die gleiche Behandlung unsern Äthylester in dieselbe Feistsche Säure überzuführen, so ist die Konstitution der oben beschriebenen Bromprodukte im Sinne der angegebenen Formeln bewiesen.

\section{Experimenteller Teil.}

Polymerisation des Formylglutaconsäureesters.

Schon bei der Darstellung des Formylglutaconsäureesters, wie sie früher beschrieben worden ist ${ }^{3}$ ), entsteht die ölige Form mit der roten Eisenchloridreaktion nebenher. Dies ist auch der Hauptgrund dafür, daß man vom verwendeten Ameisensäureester aus gerechnet nur etwa 40 Proz. der theoretischen Menge an reiner Kupferverbindung und 30 Proz. an reinem Ester erhält. Jedesmal wenn er durch Ansäuern des Natrium- oder Kupfersalzes in Freiheit gesetzt wird, verwandelt sich ein Teil in die ölige dimere Form. Diese wird beim Ausäthern oder beim Ausschütteln mit Benzol hartnäckig vom Wasser zurückgehalten. Will man also das ölige Nebenprodukt gewinnen, so muß man die durch einmaliges Ausschütteln vom gewöhnlichen Ester befreiten wäßrigen Lösungen oftmals weiter ausäthern. Auch die

1) Ber. d. d. chem. Ges. 17, 2397 (1884); diese Annalen 264, 276 (1891); 273, 173 f. (1893).

2) Ber. d. d. chem. Ges. 34, 1992 (1901).

3) W. Wislicenus, Bindemann, diese Annalen 316, 31 (1901). 
dunkelgrünen, nicht krystallisierenden Mutterlaugen, welche bei der Darstellung und der Reinigung der Kupferverbindung übrig bleiben, geben beim Ansäuern dieses Nebenprodukt. Annähernd gereinigt kann es durch Auflösen in Benzol und Ausfällen mit Petroläther werden.

Die Bedingungen, unter denen die Polymerisation erfolgt, wurden durch besondere Versuche festgestellt, bei denen der reine, aus Benzol umkrystallisierte Formylglutaconsäureester als Ausgangsmaterial diente. Die rähölige Beschaffenheit und die rote Eisenchloridreaktion sind die bequemsten Merkmale der eingetretenen Veränderung.

Bei gewöhnlicher Temperatur und im Exsiccator tritt die Umwandlung nur sehr langsam ein. Eine Probe der Krystalle des monomolekularen Esters war unter diesen Umständen erst nach 3 Wochen klebrig geworden und nach 2 Monaten auseinandergeflossen. Das Gewicht war unverändert geblieben.

Noch länger halten sich die Krystalle in einer Atmosphäre, die Benzoldampf enthält. Benzol verzögert die Polymerisation.

Dagegen ist die Anwesenheit von Feuchtigkeit ein beschleunigendes Moment. Eine Probe der Krystalle, die unter einer Glasglocke neben einem Schälchen mit Wasser stand, war schon in 2 Tagen zerflossen und hatte rote Eisenchloridreaktion angenommen. (Sie hatte dabei um etwa 7 Proz. an Gewicht zugenommen und verlor das Wasser im Exsiccator sehr langsam und erst nach wochenlangem Stehen wieder.)

Noch schneller erfolgt die Polymerisation bei Wasserbadtemperatur. Wenn man die geschmolzenen Krystalle der gewöhnlichen Form sofort wieder abkühlen läßt, so erstarrt die Masse wieder und ist unverändert. Nach einstündigem Erhitzen einer Probe trat keine Krystallisation mehr ein, aber die Eisenchloridreaktion war noch blauviolett. Nach dreistündigem Erwärmen dagegen war 
anch diese kirschrot geworden und die Umwandlung beendigt. Eine Gewichtsänderung war nicht eingetreten.

Recht glatt verläuft der Polymerisationsvorgang in feuchtem Äther. Eine etwa 5́ prozentige Lösung des krystallisierten Esters hinterläßt schon nach ein- bis zweitägigem Stehen beim Verdunsten die dimere ölige Form. Setzt man aber einer solchen Lösung zu Anfang einen Tropfen verdünnter Salzsäure zu, so bleibt sie monatelang unverändert und verliert die blauviolette Eisenreaktion nicht. Säure hindert also den UmwandlungsprozeB.

Beim Abkühlen der Schmelze oder beim Verdunsten der Lösungen bleibt der dimere Formylglutaconsäureester als zähfiussiges gelbliches, in ganz reinem Zustande aber fast farbloses Öl von Honigkonsistenz zurück. Aus dem Rohprodukt krystallisieren häufig bei längerem Aufbewahren kleine Mengen von Trimesinsäureester in feinen Nädelchen aus. In den organischen Lösungsmitteln mit Ausnahme von Petroläther ist die Substanz sehr leicht löslich.

I. $0,1314 \mathrm{~g}$ gaben $0,2684 \mathrm{CO}_{2}$ und $0,0780 \mathrm{H}_{2} \mathrm{O}$.

II. $0,1139 \mathrm{~g} \quad, \quad 0,2350 \quad \mathrm{CO}_{2} \quad, \quad 0,0690 \mathrm{H}_{2} \mathrm{O}$.

III. $0,1809 \mathrm{~g} \quad, \quad 0,3706 \quad \mathrm{CO}_{2} \quad, \quad 0,1023 \mathrm{H}_{2} \mathrm{O}$.

Ber. für

Gef.

\begin{tabular}{ccrrr} 
& $\left(\mathrm{C}_{10} \mathrm{H}_{14} \mathrm{O}_{5}\right)_{2}$ & I & \multicolumn{1}{c}{ II } & III \\
$\mathrm{C}$ & 56,1 & 55,7 & 56,3 & 55,9 \\
$\mathrm{H}$ & 6,5 & 6,6 & 6,8 & $\mathbf{6 , 3}$
\end{tabular}

I. $0,1009 \mathrm{~g}$ bzw. 0,1194 in 17,79 Benzol: Gefrp.-Ern. 0,069 ${ }^{\circ}$ bzw. $0,083^{\circ}$.

II. $0,1842 \mathrm{~g}$ in 11,38 Phenol: Gefrp.-Ern. $0,270^{\circ}$.

III. $0,1182 \mathrm{~g}, 19,12$ Benzol: $\quad 0,079^{\circ}$.

Ber. für Gef.

Mol.-Gew.

$\mathrm{C}_{20} \mathrm{H}_{98} \mathrm{O}_{10}$ 428

419 bzw. $412 \quad 431 \quad 402$

Analysen und Molekulargewichtsbestimmungen I und II sind mit Präparaten ausgeführt worden, welche durch Erhitzen des einfachen Esters polymerisiert worden waren, die Bestimmungen III dagegen mit Präparaten, bei denen die Polymerisation durch Verweilen in feuchtem Äther eingetreten war. 
Die alkoholische Lösung wird durch Eisenchlorid tiefrot gefärbt. In alkoholisch-ätherischer Kaliumäthylatlösung entsteht ein lebhaft orangegelb gefärbtes Salz, das sehr hygroskopisch ist. In Chloroformlösung wird kein Brom addiert, auch Bromwasserstoff ist ohne Wirknng; beim Kochen der ätherischen Lösung mit Phenylhydrazin wurde neben etwas Trimesinsäureester die Substanz unverändert zurückgewonnen. Weder mit Benzoylchlorid noch mit Nitrobenzoylchlorid in alkalischer Lösung trat Reaktion ein. Dagegen scheint eine Kupferverbindung zu existieren, die beim Schütteln der ätherischen Lösung mit Kupferacetat als tiefgrüne schleimige Masse ansfällt. Sie gibt eine rote Eisenchloridreaktion und wird aus Äther durch Petroläther als grünes Öl gefällt. Es war nur einmal bei Winterkälte gelungen, eine kleine Menge grüner Krystalle vom Schmelzp. $97^{\circ}$ zu erhalten.

\section{Zersetzung des dimeren Formylglutaconsüureesters beim Erhitzen.}

Die im allgemeinen Teil erwähnte Zersetzung in 'Trimesinsäureester', Ameisensäure und Essigester läßt sich am glattesten bewerkstelligen, wenn man das gut getrocknete Öl bei niederem Druck in einem Ölbad erhitzt. Zum Nachweis von Ameisensäure und Essigester wird die Destillationsvorlage durch ein Aceton-KohlendioxydKältegemisch abgekühlt. Wenn die Badtemperatur etwa $120^{\circ}$ erreicht hat, tritt eine lebhafte Siedeerscheinung ein und in der Vorlage sammelt sich eine farblose, leicht bewegliche Flüssigkeit an. Erhitzt man nun weiter, so beginnt bei etwa $200^{\circ}$ unter einem Druck von $12 \mathrm{~mm}$ die Destillation von Trimesinsäureester, der sich dabei aber stark zersetzt. Man tut deshalb gut, die Badtemperatur nicht über $140-150^{\circ}$ steigen zu lassen und den zurück= bleibenden Trimesinsäureester durch Anreiben mit Äther und Umkrystallisieren aus verdünntem Alkohol zu reinigen.

Das Destillat läßt sich durch Wiederholung der Destillation unter gewöhnlichem Druck glatt in zwei 
Fraktionen zerlegen, von denen der erste (bei $75-78^{\circ}$ aufgefangen) Essigester, die zweite $\left(100-107^{\circ}\right)$ Ameisensäure ist. Diese Zersetzungsprodukte wurden in einer Aus= beute von 90 Proz. der theoretischen erhalten, die Reaktion

$$
\mathrm{C}_{40} \mathrm{H}_{28} \mathrm{O}_{19}=\mathrm{C}_{6} \mathrm{H}_{3}\left(\mathrm{COOC}_{2} \mathrm{H}_{5}\right)_{3}+\mathrm{CH}_{3} . \mathrm{COOC}_{2} \mathrm{H}_{5}+\mathrm{HCOOH}
$$

verläuft also fast ganz glatt.

Auch der monomolekulare krystallisierte Formylglutaconsäureester gibt bei der Vakuumdestillation dieselbe Zersetzung. Dies war vorauszusehen, da er sich ja bei $80^{\circ}$ in das ölige Polymere verwandelt.

\section{Einwirkung von Phenylisocyanat auf den dimeren Formyl- glutaconsäureester.}

1 Mol. dimerer Ester wurde mit 2 Mol. Kahlbaumschem Phenylisocyanat in ein mit Säure ausgespültes, gut gereinigtes und getrocknetes Glasrohr eingeschmolzen. Von dem Isocyanat wirkte aber nur die Hälfte ein. Der Rest war zu einem kleinen Teil (offenbar infolge eines Feuchtigkeitsgehaltes des öligen Esters) in Diphenylharnstoff verwandelt, zum größeren Teil aber unverändert geblieben. Die ganze Masse wurde in Äther aufgenommen und mit Wasser geschüttelt. Dabei verwandelte sich das nnveränderte Phenylisocyanat in Diphenylharnstoff. Die ätherische Lösung hinterließ beim Abdunsten ein gelbes Öl, das durch mehrmaliges Lösen in Äther und Fällen mit Petroläther möglichst gereinigt wurde. Zur Krystallisation konnte es nicht gebracht werden. Nach längerem Verweilen im evakuierten Exsiccator wurde es analysiert.

$0,1688 \mathrm{~g}$ gaben $0,3688 \mathrm{CO}_{2}$ und $0,0976 \mathrm{H}_{2} \mathrm{O}$.

$0,3350 \mathrm{~g} \# 8,3 \mathrm{ccm}$ Stickgas bei $21^{\circ}$ und $726 \mathrm{~mm}$ Druck.

$\begin{array}{ccr} & \text { Ber. für } \mathrm{C}_{27} \mathrm{H}_{33} \mathrm{O}_{11} \mathrm{~N} & \text { Gef. } \\ \mathrm{C} & 59,2 & 59,6 \\ \mathrm{H} & 6,1 & 6,4 \\ \mathrm{~N} & 2,7 & 2,8\end{array}$

Danach hat sich zwischen einem Molekül des dimeren Formylglutaconsäureesters und einem Molekül Phenylisocyanat ein Additionsprodukt gebildet, welches mit der Muttersubstanz die Abneigung, zu krystallisieren, 
teilt. Eine Eisenchloridreaktion zeigt die Substanz nicht; wenn aber die alkoholische Lösung mit dem Eisenchlorid längere Zeit steht, so tritt allmählich eine Rotfärbung anf, die auf eine Spaltung der Substanz zurückzuführen ist. Beim Erhitzen tritt zuerst Abspaltung von Phenylisocyanat und dann die beschriebene Zersetzung in Essigester, Ameisensäure und Trimesinsäureester ein.

Acylderivate des monomolekularen Formylglutaconsäureesters.

Benzoylderivat, $\left(\mathrm{C}_{10} \mathrm{H}_{13} \mathrm{O}_{5}\right) \mathrm{CO} . \mathrm{C}_{6} \mathrm{H}_{5}$.

Am besten läßt sich der gewöhnliche krystallisierte Formylglutaconsäureester benzoylieren, wenn man ihn mit der molekularen Menge Benzoylchlorid, Äther und einem Überschuß von Natronlauge schüttelt. Es bildet sich vorübergehend ein himbeerrotes Öl. Die ätherische Lösung hinterläßt farblose Nadeln, die aus Äther umkrystallisiert bei $63,5^{\circ}$ schmelzen.

$0,1500 \mathrm{~g}$ gaben $0,3538 \mathrm{CO}_{2}$ und $0,0780 \mathrm{H}_{2} \mathrm{O}$.

$\begin{array}{ccr} & \text { Ber. für. } \mathrm{C}_{17} \mathrm{H}_{18} \mathrm{O}_{6} & \text { Gef. } \\ \mathrm{C} & 64,2 & 64,3 \\ \mathrm{H} & 5,6 & 5,8\end{array}$

Die Substanz zerfließt bei längerem Aufbewahren.

$p$-Nitrobenzoylderivat, $\left(\mathrm{C}_{10} \mathrm{H}_{13} \mathrm{O}_{5}\right) \mathrm{CO}_{0} \mathrm{C}_{6} \mathrm{H}_{4} \mathrm{NO}_{2}$.

Auf die gleiche Weise erhält man die p-Nitrobenzoylverbindung. Beim Schütteln der ätherischen Lösung von Formylglutaconsäureester und Nitrobenzoylchlorid mit Natronlauge wird letztere vorsichtig und nur bis zum Eintritt dauernder schwach alkalischer Reaktion zlgegeben. Die Substanz krystallisiert aus Äther in farblosen Nadeln vom Schmelzp. $117^{\circ}$.

$0,1519 \mathrm{~g}$ gaben 0,3131 $\mathrm{CO}_{2}$ und $0,0672 \mathrm{H}_{2} \mathrm{O}$.

$0,1696 \mathrm{~g}, \quad 6,1 \mathrm{~cm}$ Stickgas bei $21^{\circ}$ und $742 \mathrm{~mm}$ Druck.

$\begin{array}{ccr} & \text { Ber. für } \mathrm{C}_{17} \mathrm{H}_{17} \mathrm{O}_{8} \mathrm{~N} & \text { Gef. } \\ \mathrm{C} & 56,2 & \mathbf{5 6 , 2} \\ \mathrm{H} & 4,7 & 4,9 \\ \mathrm{~N} & 3,9 & 4,0 \\ & & 25^{*}\end{array}$


Eine analoge Reaktion trat bei der bimolekularen Form weder mit Benzoylchlorid noch mit Nitrobenzoylchlorid ein.

\section{Phenylisocyanatadditionsprodukt, $\left(\mathrm{C}_{10} \mathrm{H}_{13} \mathrm{O}_{5}\right) \mathrm{CO} . \mathrm{NH} . \mathrm{C}_{6} \mathrm{H}_{5}$.}

Formylglutaconsäureester wurde mit etwa dem $1 \%$ fachen der berechneten Menge Phenylisocyanat in ein reines Glasrohr eingeschmolzen. Nach einigen Stunden war völlige Lösung eingetreten; am nächsten Tage war die Masse größtenteils krystallinisch erstarrt. Nach längerem Stehen wurde das Reaktionsgemisch in Äther aufgenommen und mit Wasser durchgeschüttelt. Dabei schied sich so viel Diphenylharnstoff ab, als dem Überschuß des zugesetzten Phenylisocyanats entsprach. Die ätherische Lösung hinterließ eine Krystallmasse, die etwas durch ölige Beimengungen verunreinigt war. Durch Lösen in Äther und Zufügen von Petroläther unter starker Abkühlung ließ sich die Substanz in Form farbloser Täfelchen von rhombenförmigem Umriß erhalten. Der Schmelzpunkt lag bei $74^{\circ}$.

$0,1188 \mathrm{~g}$ gaben $0,2686 \mathrm{CO}_{2}$ und $0,0634 \mathrm{H}_{2} \mathrm{O}$.

$0,1669 \mathrm{~g}, 6,5 \mathrm{ccm}$ Stickgas bei $17^{\circ}$ und $730 \mathrm{~mm}$ Druck.

$\begin{array}{ccr} & \text { Ber. für } \mathrm{C}_{17} \mathrm{H}_{19} \mathrm{O}_{6} \mathrm{~N} & \text { Gef. } \\ \mathrm{C} & 61,3 & 61,7 \\ \mathrm{H} & 5,7 & 5,9 \\ \mathrm{~N} & 4,2 & 4,4\end{array}$

Beim Erhitzen spaltet die Substanz wieder Phenylisocyanat ab.

Diese drei Acylderivate des Formylglutaconsäureesters leiten sich ohne Zweifel von der Enolform $a b$, sind also Oxymethylenderivate. Sie zeigen in alkoholischer Lösung keine Eisenchloridreaktion. Läßt man die Proben aber stehen, so beginnt die Flüssigkeit schon nach 10 Minuten die blauviolette Färbung zu zeigen offenbar die Folge einer Abspaltung der Säurereste.

\section{Bromierung des Formylglutaconsäureesters.}

Wenn man Chloroformlösungen von Formylglutaconsäureester und von Brom zusammenfließen läßt, so 
verschwindet die Farbe des Broms, solange nicht mehr als ein Molekül Brom auf ein Molekül des Esters vorhanden ist. Dabei erwärmt sich die Flüssigkeit etwas. Beim Abdunsten des Lösungsmittels im trocknen Luftstrom hinterbleibt das Additionsprodukt als ziemlich leichtfließendes Öl, das keine Eisenchloridreaktion zeigt. Erst nach einigem Stehen der mit Eisenchlorid versetzten alkoholischen Lösung tritt allmählich eine Rotfärbung auf. Nach 24 stïndigem Verweilen im evakuierten Exsiccator wurde eine Brombestimmung ausgeführt.

$0,2168 \mathrm{~g}$ gaben $0,2187 \mathrm{AgBr}$.

$$
\begin{array}{ccc} 
& \text { Ber. für } \mathrm{C}_{10} \mathrm{H}_{14} \mathrm{O}_{5} \mathrm{Br}_{2} & \text { Gef. } \\
\mathrm{C} & 42,7 & 42,9
\end{array}
$$

Bleibt das Öl längere Zeit neben Kali im Exsiccator, so nimmt Gesamtgewicht und Bromgehalt ab: es wird Bromwasserstoff abgespalten. Rascher erfolgt die gleiche Veränderung beim Erhitzen oder Destillieren im Vakuum. Dabei konnte gelegentlich der ölige Monobromformylglutaconsänreester mit der roten Eisenchloridreaktion, oder sein krystallisierendes Isomeres mit violetter Eisenchloridreaktion bei Anwendung kleiner Mengen und rascher Destillation in Hochvakuum isoliert werden. Dieses Resultat hat man aber nicht sicher in der Hand, weil sich gleich nach der Abspaltung von Bromwasserstoff eine solche von Alkohol einstellt. Das Findresultat der Destillationen ist immer der Bromcumalinsäureester.

\section{Öliger Bromformylglutaconsäureester.}

Diese Form wurde nur einigemal erhalten, indem das Additionsprodukt im Vakuum bei $14 \mathrm{~mm}$ Druck und $180^{\circ}$ destilliert wurde. ${ }^{1}$ )

1) Es schien, als ob die Anwesenheit von etwas Bromwasserstoff zur Bildung und Erhaltung dieser Form notwendig sei, doeh gaben die Versuche, dies festzustellen, keine sicheren Anhaltspunkte. 
$0,1928 \mathrm{~g}$ gaben $0,2846 \mathrm{CO}_{2}$ und $0,0730 \mathrm{H}_{2} \mathrm{O}$.

$0,1850 \mathrm{~g}, \quad 0,1178 \mathrm{AgBr}$.

$\begin{array}{lcr} & \text { Ber. für } \mathrm{C}_{10} \mathrm{H}_{13} \mathrm{O}_{5} \mathrm{Br} & \text { Gef. } \\ \mathrm{C} & \mathbf{4 1 , 0} & \mathbf{4 0 , 2} \\ \mathrm{H} & 4,4 & 4,4 \\ \mathrm{Br} & 27,3 & 27,1\end{array}$

$0,1282 \mathrm{~g}$ in $18,99 \mathrm{~g}$ Benzol zeigten eine Gefrierpunktserniedrigung von $0,116^{\circ}$. Molekulargewicht berechnet 294, gefunden 297 .

Die Eisenchloridreaktion in alkoholischer Lösung war intensiv rot. Mit Kaliumäthylat entstand die andere Form.

\section{Fiester Bromformylglutaconsäureester, $\mathrm{COOC}_{2} \mathrm{H}_{6} . \mathrm{C}: \mathrm{CH}(\mathrm{OH})$<smiles>[CH][C+]=C(CC)C(=O)OCC</smiles>

Diesen Ester kann man leicht darstellen, wenn man das beschriebene Additionsprodukt in Äther löst und mit einer alkoholisch-ätherischen Lösung der molekularen Menge Kaliumäthylat zusammengibt. Es entsteht ein Niederschlag von Kaliumbromid. Das Filtrat wird ohne Anwendung höherer Temperatur verdunstet und hinterläßt ein rasch erstarrendes öl in nahezu quantitativer Ausbeute. Aus wenig Äther krystallisiert die Verbindung in langen farblosen Nadeln vom Schmel\%punkt $55-56^{\circ}$.

$0,1092 \mathrm{~g}$ gaben $0,1648 \mathrm{CO}_{2}$ und $0,0436 \mathrm{H}_{2} \mathrm{O}$.

$0,0926 \mathrm{~g} \quad 0,0,0597 \mathrm{AgBr}$.

$\begin{array}{lcr} & \text { Ber. für. } \mathrm{C}_{10} \mathrm{H}_{18} \mathrm{O}_{5} \mathrm{Br} & \text { Gef. } \\ \mathrm{C} & 41,1 & 41,2 \\ \mathrm{H} & 4,4 & 4,4 \\ \mathrm{Br} & 27,3 & 27,4\end{array}$

In den organischen Lösungsmitteln ist sie durchweg leicht löslich. Die alkoholische Lösung färbt sich anf Zusatz von Eisenchlorid tief violett.

Die Kupferverbindung ist ein lebhaft hellgrünes Krystallpulver, das aus Benzol in Form mikroskopisch kleiner Prismen vom Schmelzp. 144-145 krystallisiert. 
Formylglutaconsäureester und seine Isomerieverhältnisse. 383

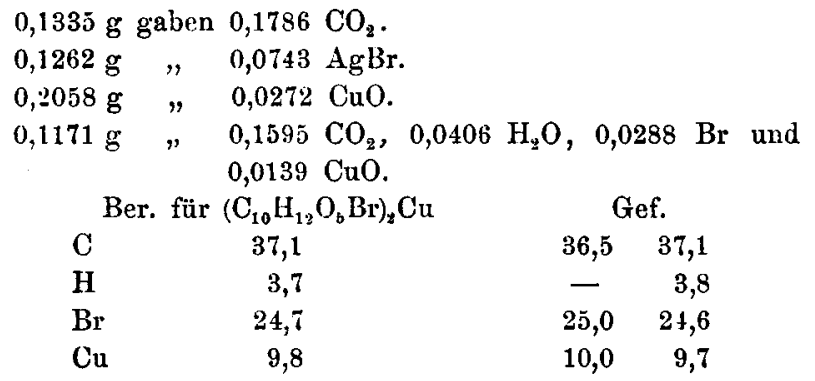

Die alkoholische Lösung gibt mit Eisenchlorid die gleiche violette Eisenchloridreaktion wie der freie Ester.

Man kann diese Kupferverbindung auch direkt aus dem Bromadditionsprodukt des Formylglutaconsäureesters darstellen, indem man direkt die bei der Bromierung erhaltene Chloroformlösuug stundenlang mit Kupferacetatlösung unter mehrmaliger Erneuerung derselben schüttelt. Auch hierbei wird Bromwasserstoff abgespalten und man erhält die Kupferverbindung in einer Ausbeute von über 80 Proz. der berechneten Menge. Durch Schütteln mit verdünnter Schwefelsäure und Äther gewinnt man daraus den freien Ester.

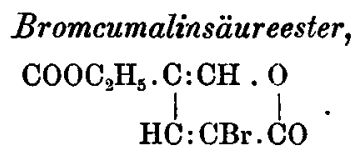

Durch Destillation des Bromformylglutaconsäureesters entsteht der Bromcumalinsäureester, aus Alkohol umkrystallisiert bildet er farbilose Prismen vom Schmelzpunkt $94-95^{\circ}$.

$0,1066 \mathrm{~g}$ gaben $0,1519 \mathrm{CO}_{2}, 0,0308 \mathrm{H}_{2} \mathrm{O}$ und $0,0808 \mathrm{AgBr}$.

$0,1064 \mathrm{~g} \quad, \quad 0,1514 \mathrm{CO}_{2}$ und $0,0288 \mathrm{H}_{2} \mathrm{O}$.

$0,1076 \mathrm{~g}, 0,0829 \mathrm{AgBr}$.

Ber. für $\mathrm{C}_{8} \mathrm{H}_{7} \mathrm{O}_{4} \mathrm{Br}$

$\begin{array}{lrrr}\mathrm{C} & \mathbf{3 8 , 9} & 38,9 & 38,8 \\ \mathrm{H} & 2,8 & 3,2 & 3,0 \\ \mathrm{Br} & 32,4 & 32,3 & 32,7\end{array}$

Durch einstündiges Kochen mit der berechneten Menge Kalilauge geht der Ester in das furfuran-2,4-di- 
384 Wislicenus u. v. Wrangell, Formylglutaconsäureester.

carbonsaure Kalium über, eine Reaktion, die für die Konstitution des Bromcumalinsäureesters und damit auch für die des Bromformylglutaconsäureesters beweisend ist. Wir fanden die Eigenschaften dieser Säure in Übereinstimmung mit den Angaben von $\mathrm{Fe}$ ist. ${ }^{1}$ )

$0,0880 \mathrm{~g}$ gaben bei $110^{\circ} 0,0092$ Gewichtsverlust.

Ber. für $\mathrm{C}_{6} \mathrm{H}_{4} \mathrm{O}_{5}+\mathrm{H}_{2} \mathrm{O}$ : Krystallwasser 10,3. Gef. 10,4 Proz.

$0,0713 \mathrm{~g}$ gaben, getrocknet, $0,1205 \mathrm{CO}_{2}$ und $0,0173 \mathrm{H}_{2} \mathrm{O}$.

Ber. für $\mathrm{C}_{6} \mathrm{H}_{4} \mathrm{O}_{5}$ Gef.

$\begin{array}{rrr}\mathrm{C} & 46,1 & 46,1 \\ \mathrm{H} & 2,6 & 2,7\end{array}$

Chlorierung des Formylylutaconsäureesters.

In eine Chloroformlösung des Formylglutaconsäureesters wurde so lange Chlor eingeleitet, bis die Gewichtszunahme einem Molekül des letzteren entsprach. Beim Verdunsten des Chloroforms erhält man ein farbloses Öl ohne Eisenchloridreaktion. Ein Entweichen von Chlorwasserstoff wurde nicht beobachtet. Bei der Destillation im Vakuum spaltet sich Alkohol ab und es entsteht eine ölige Substanz, die aufzufassen ist als der

\section{Dihydrochlorcumalinsäureester, $\mathrm{COOC}_{2} \mathrm{H}_{5}$. C : CH . $\mathrm{O}$ \\ Cl.H.C. CHCl. $\mathrm{CO}$}

Er siedet bei $175-180^{\circ}$ unter einem Druck von $18-20 \mathrm{~mm}$.

$0,1861 \mathrm{~g}$ gaben $0,2749 \mathrm{CO}_{2}$ und $0,0542 \mathrm{Cl}$.

$0,1738 \mathrm{~g} \quad, \quad 0,2513 \mathrm{CO}_{2}, 0,0574 \mathrm{H}_{2} \mathrm{O}$ und $0,0522 \mathrm{Cl}$.

$0,2579 \mathrm{~g} \quad, \quad 0,3102 \mathrm{AgCl}$.

\begin{tabular}{lccrc} 
& Ber. für $\mathrm{C}_{8} \mathrm{H}_{8} \mathrm{O}_{4} \mathrm{Cl}_{2}$ & \multicolumn{3}{c}{ Gef. } \\
C & 40,2 & 40,3 & 39,5 & - \\
$\mathrm{H}$ & 3,4 & - & 3,7 & - \\
$\mathrm{Cl}$ & 29,7 & 29,1 & 29,7 & 29,7
\end{tabular}

1) Ber. d. d. chem. Ges. 34, 1994 (1901). 